\title{
MODELING AND SIMULATING PEDESTRIAN SOCIAL GROUP BEHAVIOR WITH HETEROGENEOUS SOCIAL RELATIONSHIPS
}

\author{
Manon Prédhumeau, \\ Julie Dugdale \\ Univ. Grenoble Alpes, LIG \\ 38000 Grenoble, France \\ manon.predhumeau@univ-grenoble-alpes.fr \\ julie.dugdale@univ-grenoble-alpes.fr
}

\author{
Anne Spalanzani \\ Univ. Grenoble Alpes, Inria, \\ 38000 Grenoble, France \\ anne.spalanzani@univ-grenoble-alpes.fr
}

\begin{abstract}
The nature of the social relationship within a pedestrian group influences the group's structure and behavior and thus its entitativity (i.e. the perception of a group as a unit by other pedestrians). However, existing crowd models ignore the diversity of social relationships and have limitations in reproducing group avoidance behaviors. The proposed model is an adaptation of the social force model that addresses group social relationships. The approach is calibrated by comparing the distances and angles between members of the simulated groups with observations in real crowds. Results show that intra-group distances are a key factor in collision avoidance behavior. Simulation of collision avoidance shows that group members behavior fits better with empirical data than the original model and that individuals avoid splitting groups. By simply tuning the distribution of social relationships in the simulated crowd, the model can be used to reproduce crowd behaviors in several contexts.
\end{abstract}

Keywords: pedestrian dynamics, multi-agent simulation, social force model, proxemics.

\section{INTRODUCTION}

Social groups represent a significant part of most urban crowds. Depending on the location and time of the day, there may be more groups than individual pedestrians (Moussaïd et al. 2010). The focus of this paper is on social groups as groups composed of at least two pedestrians who intentionally move together and who have a persistent social relationship, such as friends, couples, coworkers and families. We are interested in a "classic" urban crowd of walking pedestrians, such as can be found every day on pedestrian streets or on a campus for example. This corresponds to an ambulatory crowd (Berlonghi 1995): "A crowd entering or exiting a venue, walking to or from car parks, or around the venue to use the facilities.".

Observations show that the nature of the social relationship within a group has a major influence on the group structure and its behavior, and thus its entitativity, i.e. the perception of a group as a single entity by others (Campbell 1958, Lickel et al. 2000). According to previous works, pedestrians are more reticent to break up a group whom they believe its members to be socially close, such as couples, than groups with less strong social ties, such as coworkers (Do, Haghani, and Sarvi 2016).

However, most multi-agent crowd models do not explicitly take into account social relationships within groups. When groups are integrated into a crowd model, they are usually represented in a uniform way, 
regardless of their relationship and their environment. In addition, although some models reproduce well the internal structure of the groups they have limitations regarding avoidance behavior. The goal of the work is to accurately simulate the movement of pedestrians. This article addresses two questions: how to use social relationships within groups to simulate several crowd contexts (business area, campus, shopping street)? How can we improve the simulation of group avoidance behavior while keeping their internal structure consistent?

To address the first question, modifications to the Social Force Model (SFM) of Moussaïd et al. (2010) are proposed, based on empirical observations from literature. The proposed model considers the relationships within groups and changes the social forces between group members according to their social relationships.

The second question on how to improve the simulation of group avoidance by other pedestrians may be approached by either adding a force to make pedestrians avoid splitting up groups, or by bringing members of a group closer together. These two solutions were tested and compared. A first version of the model adds a repulsive force, exerted by the groups to other agents; this represents the unwillingness of the pedestrians to split a social group. A second version of the model simply puts the groups members closer together and adds no additional force. The model is calibrated by measuring distances and angles between group members in the simulation and by comparing these measures with observations in real crowds. This calibration uses two scenarios: a business area with many coworkers, and a leisure area on Saturdays with mostly friends, families and couples. In both scenarios, six crowd densities are studied, from $0.04 \mathrm{p} / \mathrm{m}^{2}$ to $0.45 \mathrm{p} / \mathrm{m}^{2}$. The model is then validated through a comparison with collision avoidance maneuvers observed in real crowds.

This paper is organized as follows. Section 2 reviews some popular group models and their limitations. Section 3 describes the proposed adaptations of these models. Section 4 explains the simulator configuration, the scenarios and the measures for evaluating the model. The simulation results are presented in section 5. Lastly, conclusions and future work are discussed.

\section{LITERATURE REVIEW}

To simulate realistic crowds, the inclusion of social groups of pedestrians is essential. Depending on the spatial-temporal context, there may be more groups than individuals. In a public place on a work day, (Moussaïd, Perozo, Garnier, Helbing, and Theraulaz 2010) found 55\% of pedestrians were in groups while on a Saturday in a commercial street it was $70 \%$.

Initial work simulating bird flocks was proposed by Reynolds (1987) who showed that group behaviors can be represented by 3 simple rules applied to individual members. However, these rules are insufficient to reproduce pedestrian group structure. From this work, a lot of models were developed; a full review on group behavior models was done by Cheng et al. (2014). Peters and Ennis (2009) designed a rule-based model to simulate plausible behaviors of small groups from observations. They concluded that adding groups to the crowd increases the visible plausibility of the scene, if particular attention is given to the ratio of individuals to groups. However, they focused more on visible plausibility than on the correctness of simulation.

After data collection (Zanlungo and Kanda 2013), Zanlungo et al. proposed a mathematical model for groups of interacting pedestrians (Zanlungo, Ikeda, and Kanda 2014). The model assumes that when traveling in a group, pedestrians want to see their goal and also try to maintain visual contact with other group members. In his thesis, Park (2013) proposes a sophisticated model based on the Common Ground theory. The model uses group based navigation with macro and micro coordination strategies, with a focus on group cohesion and the conservation of its social space. Qiu and $\mathrm{Hu}$ (2010) simulated intra-group structure and inter-group relationships with the influence of group leaders.

One of the most famous physical force models to simulate pedestrians is the SFM (Helbing and Molnár 1998), with three forces describing the internal motivations of individual pedestrians. A desire force rep- 
resents the agent's aspiration to move toward its destination. A repulsive "social force" comes from other pedestrians and static obstacles to avoid collisions. A third force models the attraction toward certain interest points in the street. Helbing's model was then extended by Moussaïd to take into account group behaviors by adding new forces (Moussaïd et al. 2010). Firstly, in the case of a collision between group members, each colliding agent feels a repulsive force in order to avoid overlapping. Secondly, a cohesion force was added to keep the group together, so that the members do not move too far from the group's center. This cohesion force is triggered when the distance between a pedestrian and his group's center of mass passes the threshold of $\frac{N-1}{2}$ meters, with $N$ equal to the group size. Finally, a gazing force was added to maintain group formation and for groups members to maintain visual contact with each other. Thus, the angle between agents walking direction and the center of mass of the group must be a maximum of $90^{\circ}$, above which, a force makes them rotate towards their group. A limitation of this model is that all groups are represented in the same way, regardless of the social relationship within the group. Bañgate et al. (2018) implemented social attachment theory with a multi-agent system. Their model integrates social relationships (e.g. family, friends, colleagues, partners) with bond strength and social distance. However, their model focused on evacuation in disasters and not specifically on group structures in crowds.

The nature of the social relations within the group has a strong effect on the group structure (Zanlungo, Yücel, and Kanda 2016). Couples walk very close and abreast, colleagues are further apart, and friends walk more abreast than family members. In the reviewed models, as in most crowd models that integrate pedestrian groups, groups are represented in a uniform manner and there is a lack of a model that takes into account the diverse social relationships within groups.

The interactions of groups with individuals and with other groups has also been investigated. A study on individual avoidance of groups showed that, on average, sparse groups are traversed by individuals and small or dense groups are avoided as a whole (Bruneau, Olivier, and Pettre 2015). During collision avoidance, a group may temporarily split when it encounters another group, an individual or an obstacle (Singh et al. 2009, Do, Haghani, and Sarvi 2016). Using cellular automata, Seitz, Köster, and Hartmann (2011) modeled the separation and reunion of groups when avoiding a static obstacle. However, observations of crowds in videos show that groups follow specific social norms; they prefer to avoid splitting up, and they only avoid groups of the same size or larger (Karamouzas 2012). Individuals contribute more to the collision avoidance effort than groups (Do, Haghani, and Sarvi 2016); for crowds with a density between 0.4 and 0.8 people $/ \mathrm{m}^{2}$, individuals changed their direction twice as much as groups. To reproduce group avoidance, Singh et al. (2009) used crowds observations to customize an existing model with new forces for groups. They added attractor points, to hold small subgroups in formation and psychological repulsive forces to model avoidance behavior when agents are on a collision course with a group. Recently, Huang et al. (2018) also adapted the SFM by adding a repulsive force from groups to others and a coordination force between sub-groups of a large group. On a different idea, in the model proposed by Park (2013), group avoidance is an emergent effect due to the increased density of the area occupied by the group.

Few models focused on the avoidance behavior of groups by others. This has been addressed either by adding an extra force for pedestrians to avoid splitting the groups, or by bringing group members closer together. Both solutions are tested in this pape and compared in simulated groups with diverse social relations.

\section{MODEL}

In our previous work, we looked at how to simulate individuals moving in crowds in open environments (Prédhumeau, Dugdale, and Spalanzani 2019). The work adapted the SFM to take into account that pedestrians dynamically adapt their personal space and their behavior depending on the perceived density of the crowd around them. This is based on work that showed that pedestrians are more willing to be closer to each other in crowded spaces, whereas in more open spaces they will expand their personal space in order to have 
a comfortable distance from others (Frohnwieser, Hopf, and Oberzaucher 2013). The behavior of individual pedestrians was well reproduced, therefore this model is used as a basis for simulating pedestrians.

A limitation of this previous work is that groups were not taken into account. Depending on the social relations within a group, the personal space of its members will be different, e.g. couples will be closer together and work colleagues will be farther apart (Zanlungo, Yücel, and Kanda 2016). Thereby, adaptations are needed to represent social groups. Moussaïd et al. (2010) extended the SFM to take into account group behaviors, focusing on the structure of groups according to their size. However, there are two limitations of this model. First, as in most models, groups are represented in a uniform way: a group of two pedestrians will always try to maintain the same distance from each other, regardless of their relationship. Second, group avoidance behavior is not well reproduced; the surrounding agents rarely avoid groups and groups frequently split compared to empirical observations.

In this paper we incorporated the model of Moussaïd et al. (2010) into the model of Prédhumeau, Dugdale, and Spalanzani (2019), in order to represent social groups. The limitations identified in the Moussaïd model are treated. The model is presented in two parts: first, introducing different social relationships into groups; second, comparing two methods for reproducing group avoidance behavior.

\subsection{Group Behavior Model}

In the model of Moussaïd et al. (2010) some pairs of agents behave in a way that it not easily explainable. For example, one agent may pass in front of another agent, who in turn passes in front of the first agent, meaning that the agents constantly cross paths instead of walking beside each other as in real crowds. This is because the social force is too strong between agents of the same group. Indeed in Moussaid's model, group members feel the same repulsive social force from other group members as non-group members. Given the distances observed in real life between group members, pedestrians accept intrusions into their personal space if it is from a member of their own group (more or less depending on the relationship) (Wei et al. 2015). The dynamic personal space defined by Prédhumeau, Dugdale, and Spalanzani (2019) can be ignored between members of a group since is not taken into account in the calculation of forces between members of the same group. In the proposed model there is less repulsion between group members than between strangers: pedestrians prefer to approach a member of their group rather than invade a stranger's personal space. Therefore, the social force between group members is reduced by 20 . This force parameter was tuned by hand in the simulator and yielded results that fits the best compared to empirical studies.

When pedestrians belong to a group they pay particular attention to other group members. If a group member is behind, $60 \%$ of people will slow down to wait and 15\% will stop (Wei et al. 2015). Therefore in the proposed model pedestrian perceptions have been adjusted so that members of the same group can perceive other members wherever they are. Thus, if a group member is several meters behind the other members, the attraction forces in the group will still apply and the pedestrian behind will accelerate to catch up with the group, while the members in front will slow down.

The social relations within a group have a large influence on its structure. The proposed model explicitly integrates four different social relationships according to Zanlungo, Yücel, and Kanda (2016): couples, friends, families and coworkers. In the proposed model couples are necessarily groups of two people while groups of friends, coworkers and families can have more members. The thresholds at which the cohesion force and the gazing force are triggered have been customized according to the type of group. Empirical crowds observations show that couples walk very close and abreast (Zanlungo, Yücel, and Kanda 2016). Therefore in the proposed model the cohesion force is stronger for the couples than for other types of groups; the original force is multiplied by 2. Moreover, this force is now triggered when the distance between a couple member and the couple's center of mass is $\frac{(N-1)}{3}$ meters. In observations, coworkers 
are more distant than other types of groups (Zanlungo, Yücel, and Kanda 2016). In the proposed model, the cohesion force in coworkers groups is lower than for other types of groups; it is divided by 2 . In addition, this force is now triggered when the distance from a group member to the group's center of mass is $\frac{3 \times(N-1)}{4}$ meters. Finally, observations showed that friends walk generally more abreast than family members (Zanlungo, Yücel, and Kanda 2016). As the original model of Moussaïd et al. (2010) produces agents that walk abreast, this element has not been changed for friends. In the proposed model, for family groups, the gazing force is triggered when the angle between the pedestrian walking direction and the gazing direction toward the group's center is $120^{\circ}$ instead of $90^{\circ}$ for other types of groups. It allows for less rigid formation, with an agent walking in front of the other group members for example.

The adaptations of the original model of Moussaïd et al. (2010) can be summarized as follow. The social force between members of a group is reduced and the personal space is ignored between members of the same group. Members of the same group can perceive other members wherever they are. Four types of groups are defined according to social relations: couples, friends, families and coworkers; and the cohesion and gazing forces are customized according to the type of group (see Table 1).

\subsection{Group Avoidance Behavior}

A major weakness of the original model of Moussaïd et al. (2010) is the lack of entitativity. A group of 3 pedestrians, for example, is perceived as 3 independent pedestrians by other pedestrians and the groups split too often compared to observations (see section 5 for quantitative comparison). To address this issue, two methods from the literature were implemented to improve group avoidance behavior.

A first method to reproduce group avoidance behavior is used by Singh et al. (2009) and Huang et al. (2018). This method consists in adding a repulsive force to represent the psychological reticence of individual pedestrians to split a group. In addition to the social force exerted by each individual pedestrian, a repulsive force is exerted by the group towards the other pedestrians in its neighborhood. The group's center of mass is considered as the position of the group. If the group to be avoided is in the attention zone of the pedestrian performing avoidance, the group's social force is multiplied by two. If the avoiding pedestrian itself belongs to a group, there is a coordinated avoidance movement; the members of the avoiding group share the same avoidance force among themselves. This prevents one member from going to the right and the other to the left, which would cause oscillations. Unless there is an emergency avoidance, group members prefer not to split and to pass an individual or another group all together.

A second method is used by Park (2013). With this method, group avoidance is not the result of an additional force but is an emergent effect due to the increased density of the area occupied by the group. Can group avoidance behavior be reproduced simply by putting group members closer together? To answer this, we developed a second model with no additional force between groups and other pedestrians; group members were put closer by lowering the distance threshold at which the cohesion force is triggered. The distance threshold to group center of mass was lowered by $10 \mathrm{~cm}$ (see Table 1); this is the distance that better fits the used empirical data. By lowering the trigger threshold, the separation of group members is penalized. Avoidance behavior should thus be more consistent, with less group splitting than in the original model.

Table 1: Overview of distances and angle thresholds by group type for both versions of the model.

\begin{tabular}{|c|c|c|c|c|}
\hline & Friends & Couples & Family & Coworkers \\
\hline Distance threshold in first version $(\mathrm{m})$ & $\frac{N-1}{2}$ & $\frac{N-1}{3}$ & $\frac{N-1}{2}$ & $\frac{3 \times(N-1)}{4}$ \\
Distance threshold in second version $(\mathrm{m})$ & $\frac{N-1}{2}-0.1$ & $\frac{N-1}{3}-0.1$ & $\frac{N-1}{2}-0.1$ & $\frac{3 \times(N-1)}{4}-0.1$ \\
\hline Angle threshold to group center of mass $\left(^{\circ}\right)$ & 90 & 90 & 120 & 90 \\
\hline
\end{tabular}




\section{SIMULATION}

The model in section 3 was implemented using Pedsim_ros (Okal, Linder, Vasquez, Wehner, Islas, and Palmieri 2014), an open source crowd simulator that implements the SFM described by Moussaiid et al. (2010). The simulation time step is $0.04 \mathrm{~s}$. The group sizes follow a zero-truncated Poisson distribution with $\lambda=1.1$ (Moussaïd et al. 2010).

Two typical crowd scenarios are considered: a business area on week day afternoon and a leisure place on Saturday afternoon. One population is generated for each scenario: the business area is mainly composed of coworkers $(66 \%)$ with a few couples $(18 \%)$, friends $(8 \%)$ and families $(8 \%)$. The leisure population is mainly composed of friends (41\%), families (27\%) and couples (30\%), with a few coworkers (3\%). These scenarios were chosen because some empirical measures are available for similar scenarios of crowds (Moussaïd et al. 2010). However, the social relationships that compose the crowd is not specified in the observations. That is why the percentages have been arbitrarily chosen to represent two typically different populations; they can be easily configured with more data available. Since the focus is on groups, we used the same ratio of individuals to groups for both scenarios. However, in a business environment there may be more individuals than groups at the start of the work day because of the increased number of commuters.

The simulation environment contains a dispersed crowd with pedestrians crossing a square place from different directions. For both scenarios, six densities which are common in public places have been studied, from $0.04 \mathrm{p} / \mathrm{m}^{2}$ with 40 agents to $0.40 \mathrm{p} / \mathrm{m}^{2}$ with 400 agents. Each simulation case has been replicated 100 times in order to get averaged results.

Density is calculated in a central zone, thus only the interacting pedestrians are considered. For each group the same measurements were used as in Moussaïd et al. (2010), that is, the distances and angles between group members (see Figure 1). In all of the results, pedestrians $p_{1}, p_{2}, p_{3}$ and $p_{4}$ refer to the members of a group, from left to right. In observations, group sizes are rarely bigger than 3 as larger groups tend to split into subgroups (Do, Haghani, and Sarvi 2016, Zanlungo and Kanda 2013). This is why only groups of up to 4 members are considered. Groups of 2 or 3 pedestrians have distinctive geometric structures when walking: an abreast formation for pairs and an abreast or a V-like formation for triads, with the central pedestrian walking slightly behind (Zanlungo and Kanda 2013). This group structure changes with increased density in real crowds with pairs moving closer together and 3-member groups changing from an abreast to a V-like formation (Moussaïd et al. 2010, Karamouzas 2012). This pattern may not be optimal to navigate efficiently. An inverted V, with the central pedestrian forward, would be more aerodynamic since it would facilitate penetration into a counter-flow of people. However, communication, which is an essential aspect of a group, is facilitated by the $\mathrm{V}$ formation. The measurements of distances and angles between group members thus provide a good way to study the groups formation. All the measures are averaged for 5 seconds, from 8 seconds to 13 seconds from the start of the simulation to let the group structures take place.

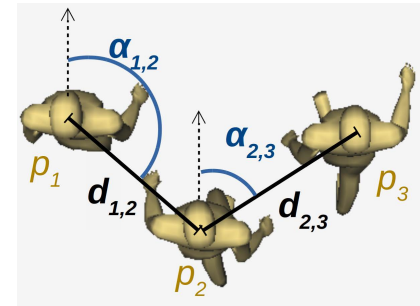

Figure 1: Measurement method from Moussaïd et al. (2010): $d_{1,2}$ and $\alpha_{1,2}$ are the distance and the angle between $p_{1}$ and $p_{2}$. Pedestrian $p_{2}$ is the closest group member on the right-hand side of $p_{1}$.

In a second simulation experiment, the avoidance maneuver performed by each type of group is studied. For each of the four group types, three avoidance scenarios are considered: one pedestrian crossing a group 
of two pedestrians, one pedestrian crossing a group of three pedestrians, and a group of two pedestrians crossing another group of two pedestrians. 20 simulations were run for each case. At the start of the simulation there is a gap of 16 meters between the groups to give them enough time to avoid the frontal collision. For each simulation, the avoidance maneuver is classified into one of three categories: avoid on the left, avoid on the right or split the group. The experiments were performed with the Moussaiid et al. (2010) original model and with the two versions of the proposed model.

\section{RESULTS AND DISCUSSION}

\subsection{Intra-Group Structure: Distances and Angles}

In the results, for the 2-member groups, the angle is constant around $90^{\circ}$ (see Table 2). Due to the gazing force, pedestrians walk side by side whatever the crowd density, as in empirical studies (Zanlungo and Kanda 2013). For the 3-member groups, the characteristic V-shape appears in both scenarios, but is slightly more accentuated in the business scenario. This is due to having a lot of coworkers in the business scenario, which have a lower cohesion force. If one agent in a coworker group is slower, the other members will wait for less time than with a family or friend group. However, they still apply a gazing force, so they will put the slowest agent in the middle to keep an optimized movement. For 4-member groups, a U-shape tends to appear, which is more accentuated in the business scenario (see Table 2).

These results were compared with empirical observations in a public place on a working day afternoon, with 1098 pedestrians, and on a Saturday afternoon in a commercial street with 3461 pedestrians in the summers of 2006 and 2007 in Toulouse, France (Moussaïd et al. 2010). Only the densities for which crowd data are available have been compared: $0.04 \mathrm{p} / \mathrm{m}^{2}$ for the business scenario and $0.25 \mathrm{p} / \mathrm{m}^{2}$ for the leisure scenario. Additional comparisons are needed to finely validate the other densities. Group formation patterns are replicated well and align with empirical studies as shown in Table 2.

Table 2: Average angles $\left(^{\circ}\right)$ between group members in observations and in the simulation for each group size, scenario and model version. Values in brackets are standard errors of the mean for observations and standard errors of the mean on 100 simulation runs.

\begin{tabular}{|c|ccc|ccc|}
\hline Group size & \multicolumn{3}{|c|}{ Business scenario } & \multicolumn{3}{c|}{ Leisure scenario } \\
\hline & $\begin{array}{c}\text { Empirical } \\
\text { observations }\end{array}$ & Version 1 & Version 2 & $\begin{array}{c}\text { Empirical } \\
\text { observations }\end{array}$ & Version 1 & Version 2 \\
\hline $2 p_{1} p_{2}$ & $89.8[ \pm 1.12]$ & $90.1[ \pm 0.4]$ & $90.2[ \pm 0.4]$ & $90.3[ \pm 0.80]$ & $90.2[ \pm 0.5]$ & $90.4[ \pm 0.4]$ \\
$3 p_{1} p_{2}$ & $97.8[ \pm 1.12]$ & $100.6[ \pm 1.0]$ & $98.5[ \pm 1.0]$ & $107.9[ \pm 2.84]$ & $95.5[ \pm 5.14]$ & $94.7[ \pm 1.1]$ \\
$3 p_{2} p_{3}$ & $87.1[ \pm 4.46]$ & $80.6[ \pm 1.1]$ & $81.4[ \pm 1.1]$ & $70.6[ \pm 2.55]$ & $84.4[ \pm 1.5]$ & $85.6[ \pm 1.6]$ \\
$4 p_{1} p_{2}$ & $99.2[ \pm 6.33]$ & $103.4[ \pm 2.5]$ & $101.8[ \pm 2.4]$ & $102.3[ \pm 5.85]$ & $103.3[ \pm 2.9]$ & $102.2[ \pm 2.9]$ \\
$4 p_{2} p_{3}$ & $87.7[ \pm 6.54]$ & $88.4[ \pm 2.4]$ & $88.0[ \pm 2.6]$ & $86.0[ \pm 4.71]$ & $81.9[ \pm 2.9]$ & $84.8[ \pm 2.5]$ \\
$4 p_{3} p_{4}$ & $85.4[ \pm 5.01]$ & $80.6[ \pm 4.5]$ & $79.5[ \pm 3.4]$ & $76.6[ \pm 5.09]$ & $79.6[ \pm 5.9]$ & $85.9[ \pm 5.2]$ \\
\hline
\end{tabular}

The intra-group distances in the business and the leisure scenarios according to crowd density are shown in Figure 2 for the first version of the model with an additional force. Overall, group members in the leisure scenario are closer than in the business scenario. This is due to a larger presence of couples, who have a stronger cohesion force and are therefore closer than other groups. Group members are farther apart in the business scenario since there is less cohesion between coworkers and this scenario includes a majority of coworkers. Members of groups of two are closer than members of larger groups, as has been observed in empirical study (Moussaïd et al. 2010). In both scenarios and for all groups sizes, when the density increases members get closer to avoid collisions with agents not in the group, as in real crowds (Karamouzas 2012). 


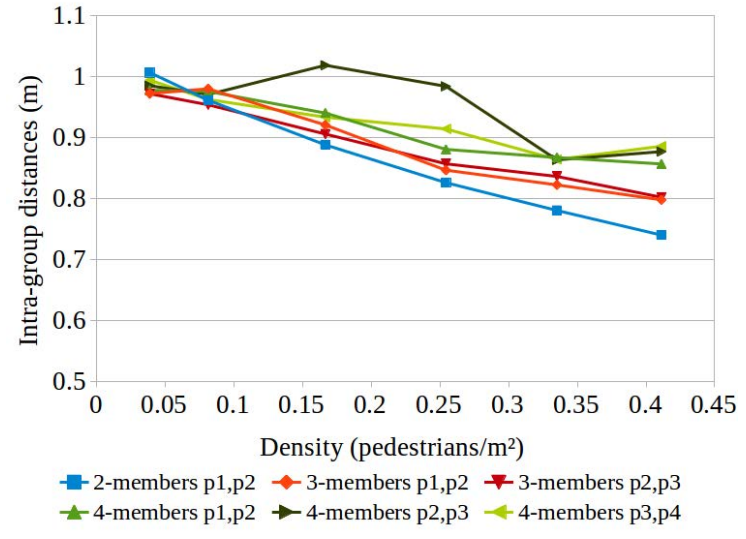

(a) Business scenario

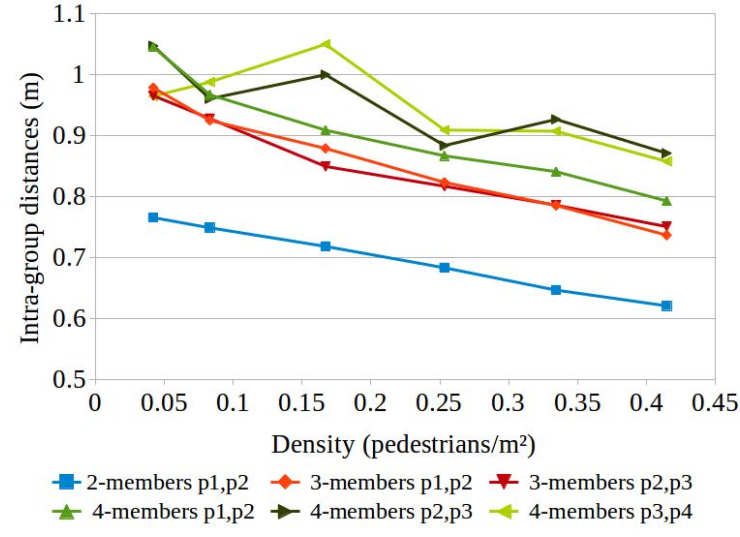

(b) Leisure scenario

Figure 2: Average intra-group distances according to the density with the first version of the model.

For the second version of the model with closer pedestrians, the intra-group distances are shown in Figure 3. As in the first model's version, group members in the leisure scenario are closer than group members in the business scenario, especially for 2-member groups. In both scenarios, and for all groups sizes the distance between group members decreases when the crowd density increases.

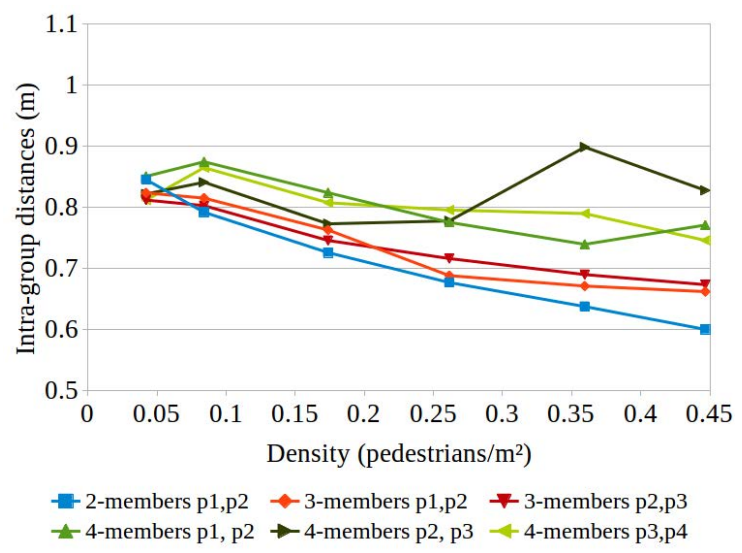

(a) Business scenario

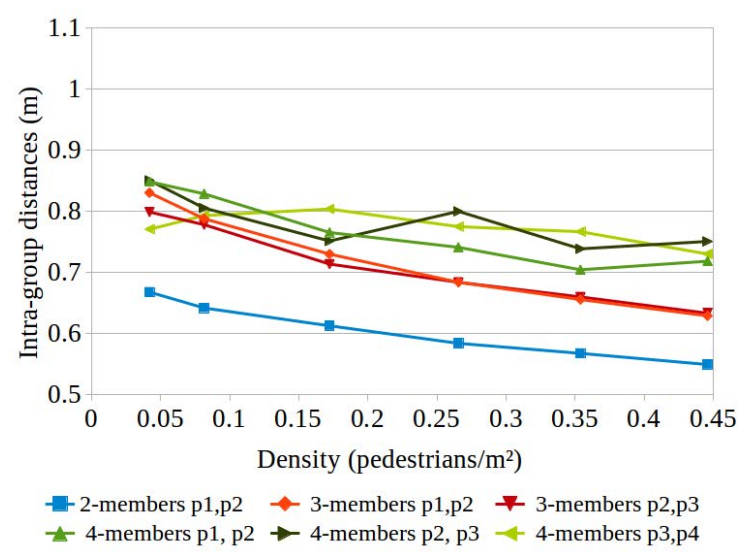

(b) Leisure scenario

Figure 3: Average intra-group distances according to the density with the second version of the model.

The two models produce close results, the shapes of the curves are similar. The main difference between the two versions is that in the second version, group members are closer. By lowering the distance threshold at which the cohesion force is triggered by $10 \mathrm{~cm}$, members of groups of two are closer by $9 \mathrm{~cm}$ on average, members of groups of three by $14 \mathrm{~cm}$ on average, and members of groups of four by $16 \mathrm{~cm}$ on average. To determine which version produces the distances that fits the most with the used empirical data, Table 3 gives a comparison between the average distances for each version and the average empirical distances from Moussaïd et al. (2010). Compared to empirical observations, the members are a little too far away in the first version. The second version, with closer agents, reproduces distances better than the first version in 11 out of 12 cases (Table 3 ).

To summarize, incorporating various social relationships within pedestrian groups allows for varying behaviors while keeping the group configurations very close to real crowd observations. The observed formations 
Table 3: Average distances (m) between group members in observations and in the simulation for each group size, scenario and model version. Values in brackets are standard errors of the mean for observations and standard errors of the mean on 100 simulation runs.

\begin{tabular}{|c|ccc|ccc|}
\hline Group size & \multicolumn{3}{|c|}{ Business scenario } & \multicolumn{3}{c|}{ Leisure scenario } \\
\hline & $\begin{array}{c}\text { Empirical } \\
\text { observations }\end{array}$ & Version 1 & Version 2 & $\begin{array}{c}\text { Empirical } \\
\text { observations }\end{array}$ & Version 1 & Version 2 \\
& & & & & \\
\hline $2 p_{1} p_{2}$ & $0.78[ \pm 0.02]$ & $1.01[ \pm 0.007]$ & $0.85[ \pm 0.007]$ & $0.54[ \pm 0.01]$ & $0.68[ \pm 0.002]$ & $0.58[ \pm 0.002]$ \\
$3 p_{1} p_{2}$ & $0.79[ \pm 0.05]$ & $0.97[ \pm 0.02]$ & $0.82[ \pm 0.02]$ & $0.55[ \pm 0.01]$ & $0.82[ \pm 0.006]$ & $0.68[ \pm 0.005]$ \\
$3 p_{2} p_{3}$ & $0.81[ \pm 0.10]$ & $0.97[ \pm 0.02]$ & $0.81[ \pm 0.02]$ & $0.62[ \pm 0.04]$ & $0.82[ \pm 0.007]$ & $0.68[ \pm 0.006]$ \\
$4 p_{1} p_{2}$ & $0.87[ \pm 0.06]$ & $0.98[ \pm 0.1]$ & $0.85[ \pm 0.05]$ & $0.67[ \pm 0.02]$ & $0.87[ \pm 0.02]$ & $0.74[ \pm 0.02]$ \\
$4 p_{2} p_{3}$ & $0.93[ \pm 0.09]$ & $0.99[ \pm 0.05]$ & $0.82[ \pm 0.04]$ & $0.66[ \pm 0.02]$ & $0.88[ \pm 0.03]$ & $0.80[ \pm 0.03]$ \\
$4 p_{3} p_{4}$ & $0.80[ \pm 0.05]$ & $0.99[ \pm 0.08]$ & $0.81[ \pm 0.06]$ & $0.64[ \pm 0.03]$ & $0.91[ \pm 0.04]$ & $0.78[ \pm 0.03]$ \\
\hline
\end{tabular}

(abreast, V-shape, U-shape), given by angles, are very similar in both scenarios and are well reproduced in both versions. In terms of distances, the group members of the leisure scenario are closer than those of the business scenario. In both scenarios, pedestrians move closer when the crowd becomes dense. With the first version, group members are too far away compared to empirical observations, while the second version, with closer member groups, reproduces well the observed distances. It is worth pointing out that the data used were collected in France and that values may vary across countries.

\subsection{Avoidance Behavior}

Three avoidance scenarios are considered: one pedestrian crossing a 2-pedestrian group (1 vs.2), one pedestrian crossing a 3-pedestrian group (1 vs.3), and a 2-pedestrian group crossing another group of two pedestrians ( 2 vs.2). This experiment is performed with the Moussaid et al. (2010) model and with the two new versions of the proposed model. The proportion of group splitting for each type of group is shown in Table 4 . In the model of Moussaïd et al. (2010), groups are rarely avoided; groups are split in $78 \%$ of the cases on average (Table 4), which is slightly unrealistic. In the first version of the proposed model, the addition of a repulsive force from groups toward surrounding pedestrians increases group avoidance; groups are split in $32 \%$ of cases (Table 4). In the second version, with closer group members, groups are split in $36 \%$ of cases (Table 4). By lowering the trigger threshold, the separation of groups is penalized: if they start to split, the force will be triggered.

Table 4: Percentage of splitting behavior in groups in simulation for each scenario and version of the model.

\begin{tabular}{|c|c|c|c|c|c|c|c|c|c|c|c|}
\hline Scenario & $\begin{array}{c}\text { Moussaïd's } \\
\text { model }\end{array}$ & Friends & Couples & $\begin{array}{l}\text { Version } \\
\text { Family }\end{array}$ & 1 & Avg. & Friends & Couples & $\begin{array}{l}\text { Version } \\
\text { Family }\end{array}$ & $\begin{array}{l}2 \\
\text { Coworkers }\end{array}$ & Avg. \\
\hline 1 vs. 2 & $75 \%$ & $15 \%$ & $0 \%$ & $0 \%$ & $100 \%$ & $29 \%$ & $25 \%$ & $0 \%$ & $0 \%$ & $100 \%$ & $31 \%$ \\
\hline 1 vs. 3 & $100 \%$ & $30 \%$ & - & $70 \%$ & $100 \%$ & $67 \%$ & $45 \%$ & - & $75 \%$ & $100 \%$ & $73 \%$ \\
\hline 2 vs. 2 & $60 \%$ & $0 \%$ & $0 \%$ & $0 \%$ & $35 \%$ & $9 \%$ & $10 \%$ & $0 \%$ & $10 \%$ & $30 \%$ & $13 \%$ \\
\hline Average & $78 \%$ & & & $32 \%$ & & & & & $36 \%$ & & \\
\hline
\end{tabular}

The percentages obtained with the proposed model are consistent with experimental observations. In Southern Cross train station in Melbourne, for a density of $0.43 \mathrm{p} / \mathrm{m}^{2}$, a $36 \%$ split was observed in groups of two, while in our model we observe $29 \%$ and $31 \%$ for 1 vs. 2 and $9 \%$ and $13 \%$ for 2 vs.2 (Table 4). In Melbourne observations, no details are given on whether it was one or two pedestrians who divided the groups of two. 
They also noticed that couples or pair friends were less likely to split. With both versions of our model, couples split less than friends and families, who split less than coworkers. Another experiment with 2member groups on a sidewalk and a single person walking toward them showed that $39 \%$ of the groups split (Knowles 1972). In our versions the results were 29\% (version 1) and 31\% (version 2). Although there is some difference between the empirical study and our results, ours are closer than Moussaïd's model where the result was $75 \%$. In the Melbourne case, more than $80 \%$ of groups of three were split (Do, Haghani, and Sarvi 2016). In our model, groups of three split more than groups of two when crossing a single pedestrian; $67 \%$ instead of $29 \%$ with the first version and $73 \%$ instead of $31 \%$ in the second version (Table 4).

In the same way, according to video observation of crowds in a university campus, Singh et al. (2009) reported $22 \%$ of split in groups during a frontal collision, without specifying group size and density.

There are some anomalies in the results. Groups of friends and families never split in the 2 vs. 2 scenario with the first version. This is improved in the second version, with $10 \%$ split. This may be close to reality as the two groups on a collision course will make an effort to avoid the other group and to stay together. In addition, couples never split in the simulation, which is slightly unrealistic. Conversely, simulated coworkers always split when they encounter a pedestrian. Since coworkers are considered to be more distant, they may prefer to split than make a detour. Experiments considering the splitting behavior by type of group needs to be further investigated. However, there is a lack of empirical data concerning this aspect that could be used to compare our results. Finally, in the simulation individuals take more responsibility for avoiding groups (see Video 2019). This result has been observed in real crowds where individual pedestrians perform more trajectory changes than groups (Do, Haghani, and Sarvi 2016).

\section{CONCLUSION}

This paper proposes modifications to the model of Moussaïd et al. (2010) to address two questions. The first is: how to use social relationships within groups to simulate various crowd contexts? To address this, the social relations within groups (couples, friends, families and coworkers) each with different social forces, are modeled. The second question is: how can we improve the simulation of group avoidance behavior? To answer this question, two versions of the model are compared. The first adds a force to represent the repulsion of pedestrians to split social groups. In the second version, no additional force is used, the group members are simply closer. The model is calibrated with two common scenarios with densities ranging from $0.04 \mathrm{p} / \mathrm{m}^{2}$ to $0.40 \mathrm{p} / \mathrm{m}^{2}$ : a business area with coworkers and a leisure place with friends, families and couples. The model is validated through a comparison with behaviors observed in real crowds.

The results show that with both versions, the avoidance behavior is closer to observed behavior than with the original model. In the collision avoidance maneuvers, pedestrians in groups have a consistent behavior and individuals avoid splitting groups. In the second version, the intra-group distances better fit observations and the avoidance behavior is very similar to the first version. This result shows that the model is very sensitive to intra-group distance, which is of major importance in avoidance behavior. Putting simulated groups members closer fits better with the distances in the used empirical data and is sufficient to reproduce most avoidance behaviors. In addition, it is easier to calibrate an intra-group distance than a psychological force, which is difficult to estimate. By varying the distance between groups members, the model integrates the concept of entitativity: collision avoidance behaviors vary according to the group's social relation. The results show that by simply adjusting the distribution of each social relationship in the simulated crowd, the model can simulate many scenarios and more scenarios will be explored in future work. The model can be used as a basis to study groups during crises and emergencies, as well as changes in the mood of the crowd.

Further work is envisaged. A focus on the particular case of children could be interesting, considering their sometimes erratic behavior. In addition, the work is currently being applied to a practical situation of socially-aware mobile robots. The robot's predictions of pedestrian groups integrates social behaviors: e.g. 
do not pass through a strongly socially cohesive group. The method recently proposed by Yucel et al. (2019) to automatically recognize social relationships within pedestrian dyads can be combined with our work on reproducing social relations in simulation to improve robot's predictions of groups in real crowds.

\section{ACKNOWLEDGMENTS}

This research is part of the HIANIC project (Human Inspired Autonomous Navigation In Crowds), funded by the French Ministry of Education and Research and the French National Research Agency.

\section{REFERENCES}

Bañgate, J., J. Dugdale, E. Beck, and C. Adam. 2018. "A multi-agent system approach in evaluating human spatio-temporal vulnerability to seismic risk using social attachment". WIT Transactions on Engineering Sciences vol. 121, pp. 47-58.

Berlonghi, A. E. 1995. "Understanding and planning for different spectator crowds". Safety Science, pp. 239-247.

Bruneau, J., A.-H. Olivier, and J. Pettre. 2015. "Going Through, Going Around: A Study on Individual Avoidance of Groups". IEEE Transactions on Visualization and Computer Graphics vol. 21 (4), pp. 520-528.

Campbell, D. T. 1958. "Common Fate, Similarity, and Other Indices of the Status of Aggregates of Persons as Social Entities". Behavioral Science vol. 3 (1), pp. 14-25.

Cheng, L., R. Yarlagadda, C. Fookes, and P. K. Yarlagadda. 2014. "A review of pedestrian group dynamics and methodologies in modelling pedestrian group behaviours". World Journal of Mechanical Engineering vol. 1 (1), pp. 2-13.

Do, T., M. Haghani, and M. Sarvi. 2016. "Group and Single Pedestrian Behavior in Crowd Dynamics". Transportation Research Record: Journal of the Transportation Research Board (2540), pp. 13-19.

Frohnwieser, A., R. Hopf, and E. Oberzaucher. 2013. "Human Walking Behavior - The Effect of Pedestrian Flow and Personal Space Invasions on Walking Speed and Direction”. Human Ethology Bulletin, pp. 9.

Helbing, D., and P. Molnár. 1998. "Social force model for pedestrian dynamics". Physical review. E, Statistical physics, plasmas, fluids, and related interdisciplinary topics vol. 51 (5), pp. 4282-4286.

Huang, L., J. Gong, W. Li, T. Xu, S. Shen, J. Liang, Q. Feng, D. Zhang, and J. Sun. 2018. "Social Force Model-Based Group Behavior Simulation in Virtual Geographic Environments". ISPRS International Journal of Geo-Information vol. 7 (2), pp. 79.

Karamouzas, I. 2012. Motion Planning for Human Crowds from Individuals to Groups of Virtual Characters. $\mathrm{Ph}$. D. thesis, Utrecht University, the Netherlands.

Knowles, E. 1972. "Boundaries around social space: Dyadic responses to an invader.". Environment and Behavior vol. 4, pp. 437-445.

Lickel, B., D. L. Hamilton, G. Wieczorkowska, A. Lewis, S. J. Sherman, and A. N. Uhles. 2000. "Varieties of groups and the perception of group entitativity.". Journal of Personality and Social Psychology vol. 78 (2), pp. 223-246.

Moussaïd, M., N. Perozo, S. Garnier, D. Helbing, and G. Theraulaz. 2010. "The Walking Behaviour of Pedestrian Social Groups and Its Impact on Crowd Dynamics". PLoS ONE vol. 5 (4), pp. e10047.

Okal, B. and Linder, T. and Vasquez, D. and Wehner, S. and Islas, O. and Palmieri, L. 2014. "GitHub repository srl-freiburg/pedsim_ros”. https://github.com/srl-freiburg/pedsim_ros. Accessed Dec. 11, 2019. 
Park, S. I. 2013. Modeling Social Group Interactions for Realistic Crowd Behaviors. Ph. D. thesis, Faculty of Virginia Polytechnic Institute and State University, Blacksburg, Virginia.

Peters, C., and C. Ennis. 2009. "Modeling Groups of Plausible Virtual Pedestrians". IEEE Computer Graphics and Applications vol. 29 (4), pp. 54-63.

Prédhumeau, M., J. Dugdale, and A. Spalanzani. 2019. "Adapting the Social Force Model for Low Density Crowds in Open Environments". In Proceedings of Social Simulation Conference 2019.

Qiu, F., and X. Hu. 2010. "Modeling group structures in pedestrian crowd simulation". Simulation Modelling Practice and Theory vol. 18 (2), pp. 190-205.

Reynolds, C. W. 1987. "Flocks, Herds, and Schools: A Distributed Behavioral Model". Computer Graphics, 21(4) vol. 4 (21), pp. 25-34.

Seitz, M., G. Köster, and D. Hartmann. 2011. "On Modeling the Separation and Reunion of Social Groups". In Emergency Evacuation of People From Buildings, pp. 7. Warsaw, Poland.

Singh, H., R. Arter, L. Dodd, P. Langston, E. Lester, and J. Drury. 2009. "Modelling subgroup behaviour in crowd dynamics DEM simulation". Applied Mathematical Modelling vol. 33 (12), pp. 4408-4423.

Video 2019. "Video illustrating the results". https://youtu.be/xVSYNc5tkdA. Accessed Mar. 11, 2020.

Wei, X., W. Lv, W. Song, and X. Li. 2015. "Survey study and experimental investigation on the local behavior of pedestrian groups". Complexity vol. 20 (6), pp. 87-97.

Yucel, Z., F. Zanlungo, C. Feliciani, A. Gregorj, and T. Kanda. 2019. "Identification of social relation within pedestrian dyads". PLOS ONE vol. 14 (10), pp. e0223656.

Zanlungo, F., T. Ikeda, and T. Kanda. 2014. "Potential for the dynamics of pedestrians in a socially interacting group". Physical Review E vol. 89 (1), pp. 012811.

Zanlungo, F., and T. Kanda. 2013. "Do walking pedestrians stabily interact inside a large group? Analysis of group and sub-group spatial structure". In Proceedings of the Annual Meeting of the Cognitive Science Society.

Zanlungo, F., Z. Yücel, and T. Kanda. 2016. "The effect of social roles on group behaviour". In Proceedings of the 8th International Conference on Pedestrian and Evacuation Dynamics (PED2016). Hefei, China.

\section{AUTHOR BIOGRAPHIES}

MANON PRÉDHUMEAU is a PhD student at University Grenoble Alps, working on modeling and simulating pedestrian behaviors in crowds. Her research is applied to the social navigation of an autonomous vehicle in shared spaces with pedestrians. Her email address is manon.predhumeau @ univ-grenoble-alpes.fr.

JULIE DUGDALE is the head of the HAwAI (Human Aware Artificial Intelligence) research team at the Grenoble Informatics Lab and an Associate Professor at University Grenoble Alps, France. Her work concerns modeling and simulating human behavior using an agent-based approach, with a focus on emergency and crisis management. Her email address is julie.dugdale@ univ-grenoble-alpes.fr.

ANNE SPALANZANI is an Associate Professor at University Grenoble Alps and is part of the Chroma (Cooperative robots adapted to human presence in environments) team at the National Institute for Research in Computer Science and Automation (Inria), France. She is the leader of the HIANIC project and her research focuses on safe navigation of robotic systems in dynamic and human populated environments. Her email address is anne.spalanzani@univ-grenoble-alpes.fr. 